\title{
El espacio público visto por los niños y los jóvenes
}

The public space being watched by children and youngsters

Diana Wiesner Ceballos* Alejandro Galante Zapata** Adriana Ayala Posso ${ }^{* * *}$

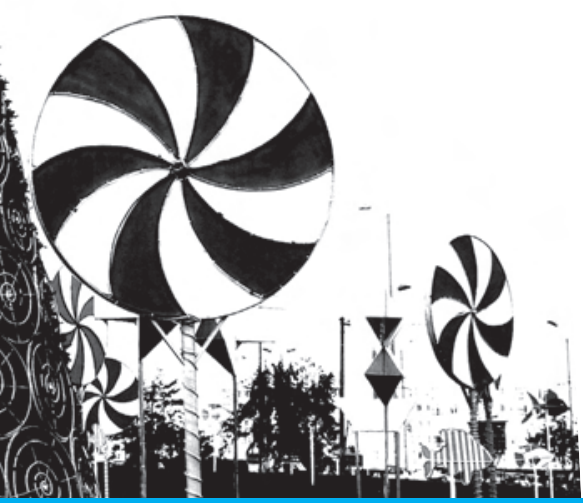

Urbanismo, Universidad Central de

Arquitecta del Paisaje, Universidad de Buenos Aires; Planea
Venezuela; profesora en la Universidad de los Andes, Bogotá.

** Estudiante de segundo semestre de arquitectura, Universidad de los Andes, Bogotá.

*** Licenciada en Educación, Especialista en Pedagogía y Docencia Universitaria, Maestría en Educación, docente. 
Resumen El presente artículo resume parte de la investigación realizada para el IDEP con el objetivo de mostrar la relación que une a los derechos colectivos, entre los cuales el espacio público integra otros como el patrimonio cultural, medio ambiente y ética pública, con la "pedagogía ciudadana" y con la formación de los niños y adolescentes en edad escolar. Se resalta la importancia de la comprensión integral del concepto de espacio público, que involucra no solamente su función de configurador de la ciudad sino también la de fusión sociocultural, ambiental, histórica y simbólica.

Dentro de él se ven claramente tres componentes: el espacial y funcional, que habla de su estructura y composición; los valores patrimoniales tangibles e intangibles, y los valores éticos — de comportamiento adecuado con el lugar y con los otros- El propósito es el de enseñarle a los niños estos temas, con su grado de complejidad según su escolaridad, mediante actividades lúdicas en espacios públicos, conociendo sitios, dibujando, realizando ejercicios sobre propuestas hechas por ellos, de manera que los concientice sobre valores éticos más complejos; estos ejemplos se ven en varios lugares del mundo, una nueva forma de enseñar a través del juego, la pintura, la literatura, el juego de actuaciones.

\section{Palabras claves: espacio público, patrimonio, medio ambiente, ética, pedagogía ciudadana, formación de los niños en edad escolar.}

Abstract This article summarizes part of the investigation realized for the IDEP with the objective to show the relation between the collective rights, in which the public space integrate others like the cultural patrimony, environment and public ethics, with the "citizen pedagogy" and with the education of boys, girls and adolescents in scholar period. The importance of integrally understanding the concept of public space is emphasized and shows that it involves not only its function of builder of the city but also as a cultural, environmental, historical and symbolic function.

Three components settle down clearly: first of all, the space and functionality which speaks of the structure and composition of the public space, the tangible and intangible patrimonial values, the ethical values, and the adequate behavior with it surrounds and to others. The purpose is to teach the children these subjects with the level of complexity according to its schooling; through playful activities in public spaces, discovering new places, drawing, realizing exercises towards new ideas and until it gives them a conscience of better ethical values. These examples are seen in several places in the world as a new way of teaching through games, painting, literature, and role plays.

\section{Key words: public space, patrimony, environment, ethics, citizen pedagogy, formation of scholars boys and girls.}




\section{Presencia del espacio público en}

\section{la escuela: la ciudad y los espacios públicos, aulas de nuevos ciudadanos}

A través de los siglos el espacio público ha incidido tácitamente en la vida de todos en lo afectivo, lo histórico, lo político, lo cultural, lo social y lo recreativo.

Parques, avenidas, callejones y plazas son lugares donde se desenvuelve la Historia, suceden historias afectivas y amorosas, se discuten ideologías y se mueven siluetas que con movimientos acompasados silenciosamente se comunican entre sí. El espacio público es un lugar que convoca a unos y otros a vivir juntos bajo el principio de equidad.

Por lo anterior, se hace importante en ciudades como la nuestra acercar a los niños y jóvenes al espacio público, con el fin de que sea el vehículo que haga posible una mejor relación consigo mismos, con su entorno y con los otros, en el marco de los derechos colectivos. Es evidente que, a través de un ejercicio pedagógico, mediante un proceso de enseñanza-aprendizaje intencionado, dialéctico y participativo, se podrá comprender que cada lugar es una oportunidad para valorarse, reconocer al otro y generar pertenencia en un tiempo determinado, cargando de significado lo que nos constituye como sujetos históricos, sociales y culturales, y dándonos la oportunidad de trascender gracias a la identidad construida.

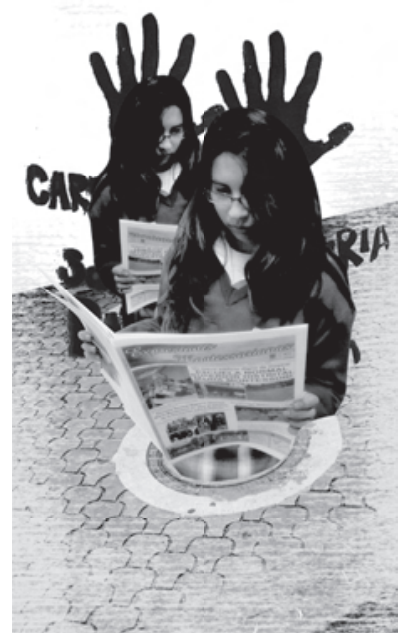

En el mundo cada día se hace más evidente la existencia de problemas de diversa índole; entre los más importantes y reiterativos están los relativos a la violencia y el deterioro de la calidad del medio ambiente, los cuales se pueden asociar de manera directa con los valores sociales y culturales básicos de la sociedad y que, por razones de procesos urbanos en los que los intereses individuales priman sobre los generales, han hecho que éstos se hayan ido perdiendo. La paulatina ausencia de valores como la solidaridad, la equidad, el respeto por las personas y por el medio ambiente, la interculturalidad, entre otros, han hecho del mundo de hoy un mundo intolerante y desinteresado. Adicionalmente, el enfoque de crecimiento de las ciudades ha dado históricamente prioridades diferentes al fortalecimiento de la familia, la comunidad y el barrio. Sólo hasta ahora se empieza a reflexionar sobre el enfoque de estructurar una ciudad sobre la base de lo público y de la calidad del medio físico; anteriormente, por el afán de dar solución a necesidades básicas, no se pensaba en la calidad de vida.

Resulta necesario tener en cuenta que esta clase de problemas sociales y culturales se dan en todo el mundo, pero en países como Colombia el problema se magnifica dado que las diferencias sociales y la desigualdad son 
mayores; los recursos y la infraestructura menores, y la necesidad ha llevado a las personas a actuar sin pensar en el otro. Adicionalmente, hay una carga cultural de Occidente tendiente al capitalismo, al consumo y a la competencia entre individuos, a diferencia de Oriente, en donde tradicionalmente los procesos se basan en el trabajo común, el respeto por el trabajo del otro y la necesidad de construir colectivamente.

Estos antecedentes hacen énfasis en la importancia y necesidad de aplicar una metodología pedagógica más participativa, recursiva y lúdica, de los procesos urbanos, mediante la cual sea posible enseñar y promover una serie de valores que hagan de las futuras generaciones de ciudadanos colombianos personas con altos índices de cultura ciudadana.

El espacio público generalmente es comprendido desde una visión urbanística y física, y definido como una “dimensión estructurante y articuladora de los sistemas urbanos y territoriales"1. Por lo tanto, es de suma importancia resaltar que el espacio público no puede tener una definición ni visión únicas. En razón del punto de vista y del saber desde donde se aborde la discusión, tiene tantos componentes que debe ser contemplado de manera integral y sobre todo humanista, de ahí que haya surgido un gran número de visiones sobre el espacio público, incluso opuestas, o que rescatan elementos antagónicos de él.

Un ejemplo es Alexander von Humboldt, quien desde su visión de naturalista lo entendió a partir de los elementos naturales, por lo cual los espacios públicos de las ciudades se debían caracterizar por la conservación y el respeto tanto de la flora como de la fauna original, cuestionando la integralidad del concepto actual del Plan Maestro de Espacio Público. Hay también quienes, como la antropóloga Mónika Therrien, consideran el espacio público como la manifestación de la nostalgia por el paisaje, refiriéndose a que en las ciudades modernas los paisajes verdes y abiertos han ido desapareciendo para darle paso a construcciones de todo tipo.

Desde lo sociológico, en cambio, el espacio público es entendido como el lugar donde los individuos tienen la libertad de establecer contacto, o distanciarse. De esta manera, son características del espacio público una integración parcial, un sistema social abierto y la interacción entre desconocidos (Bahrdt, 1969).

A partir de entonces es posible evidenciar unas primeras aproximaciones al espacio público que se alejan en gran medida de lo estrictamente físico, lo que ya nos hace pensar que tal vez los elementos constitutivos de este espacio hagan referencia a categorías diferentes a las estrictamente urbanísticas y estén más relacionados con temas de índole social, política, ecológica, histórica y cultural.
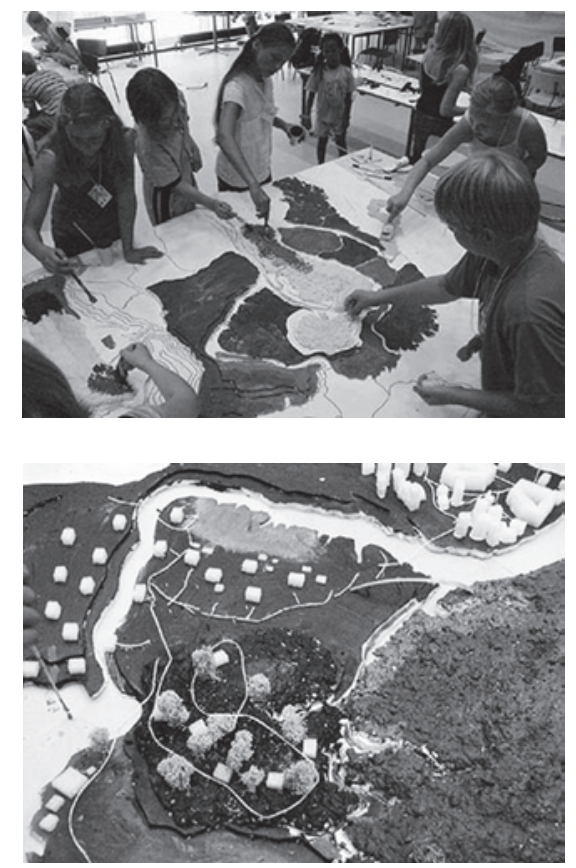

1 Decreto 215 de 2005, artículo 3. 
Así, es posible identificar también a profesionales como el arquitecto argentino Horacio Gabriel Pucheta Lascano, quien no da una definición muy específica ni literal sobre espacio público, sino que, por el contrario, se encarga de descomponer dicho espacio hasta develar cada uno de los elementos que lo componen, encontrando, en espacios como los parques, lugares en los cuales las personas se relacionan con otros y con la naturaleza.

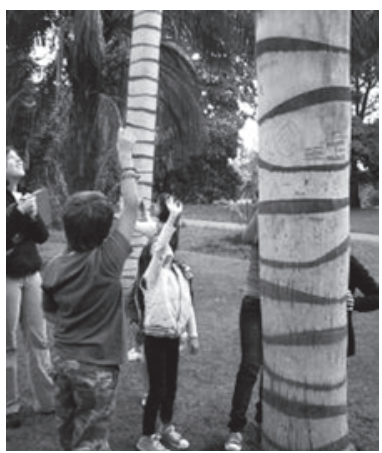

Ejercicios de Educación Medioambiental Jardín Botánico. Programa "Diseñando", Universidad de los Andes.

En el mundo se están dando ejemplos muy interesantes sobre el aprendizaje del espacio público mediante la experimentación, el juego, la literatura y el arte. Dicho aprendizaje se puede lograr en los mismos espacios urbanos donde los niños son protagonistas, ya que la interacción permite exponer sus comprensiones acerca de sus funciones, usos, cuidados y valor simbólico. Los niños son capaces de proponer, crear, transformar y administrar lugares desde su propia perspectiva. Este proceso resulta vital implementarlo en los niños y adolescentes para iniciar su proceso de cultura ciudadana, en busca de un mañana con mujeres y hombres más conscientes, participativos y éticos con los lugares y con los otros.
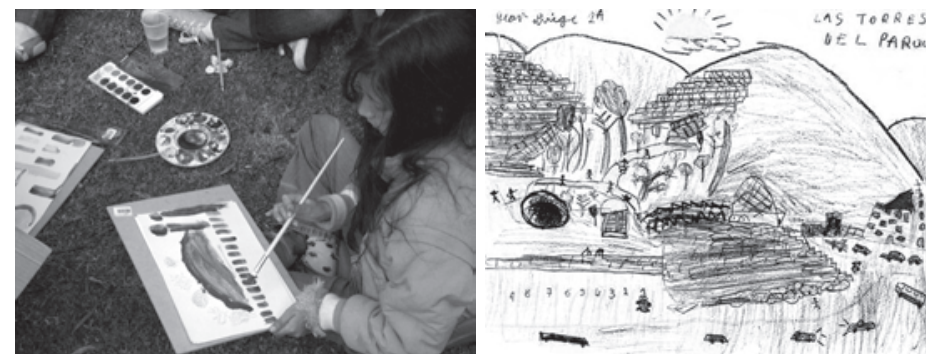

Dibujos de niños de $2^{\circ}$ grado en las Torres del Parque. Programa "Diseñando", Universidad de los Andes.

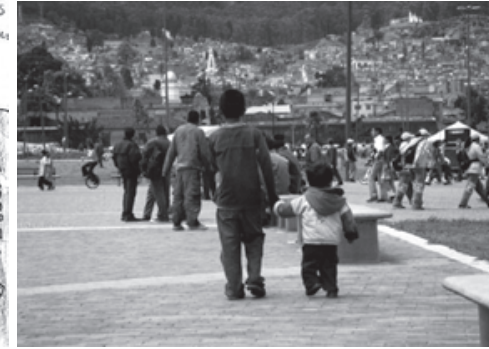

Parque Tercer Milenio, Bogotá. 


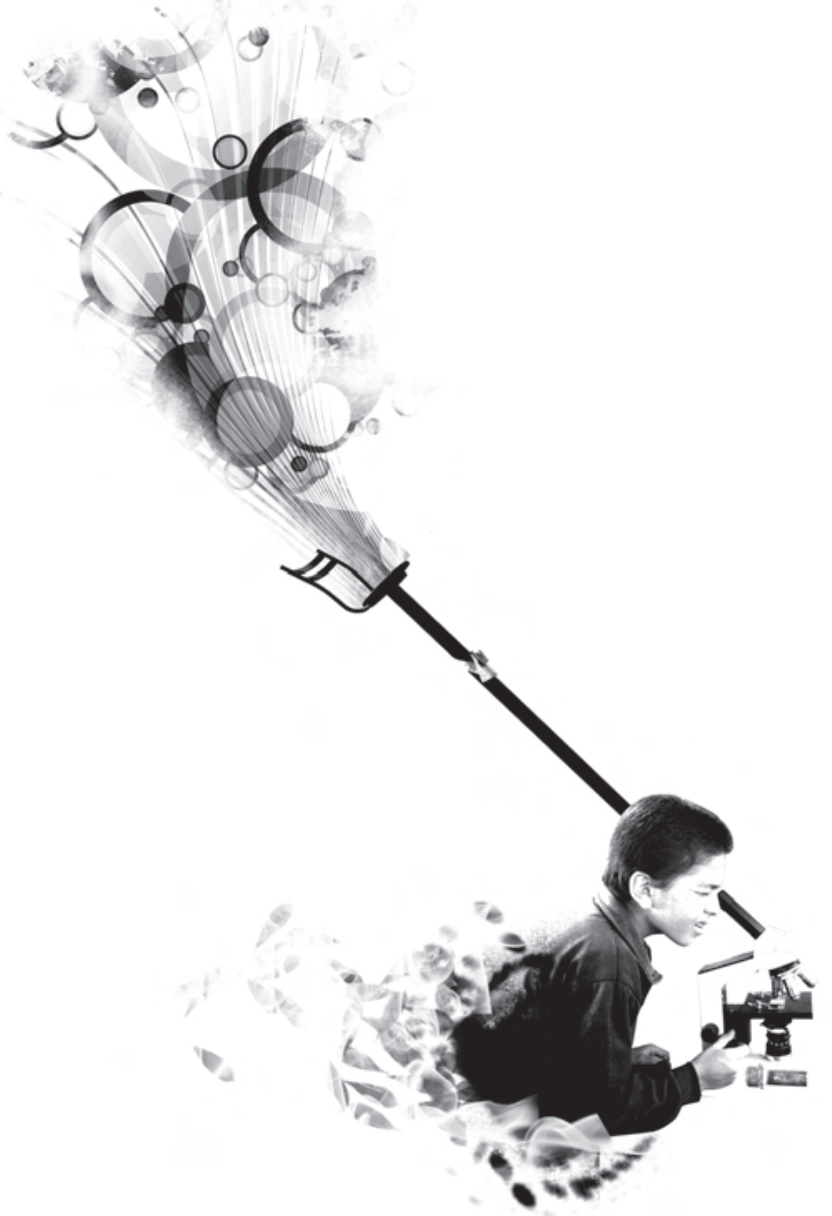

La ciudad es un escenario de aprendizaje fundamental y debe convertirse en aula de niños, jóvenes y adultos. Es vital que la visión individualista se transforme y tengamos ciudadanos más participativos, conscientes, defensores de los medioambientes e involucrados en la transformación humana de la ciudad.

En este proceso se dan los acuerdos ciudadanos y los equilibrios y equidades que permiten la vida comunal y restablecer el valor de la familia; lugar de encuentros y desencuentros ${ }^{2}$ que le brinda a sus moradores identidad como grupo social ${ }^{3}$.

Como se mencionó, resulta fundamental enfatizar que su comprensión involucra tantos componentes del comportamiento humano que, convertir el espacio público en aulas de aprendizaje sobre la relación con el medio natural y urbano y con los otros, aumentará los valores de equidad, interculturalidad, tolerancia, entre otros, y que, a futuro, esas generaciones, contagiadas de una nueva visión de la sociedad, construirán unas ciudades más amables, gozarán de espacios más estimulantes y llenos de naturaleza con sentido de pertenencia, y reirán entre amigos del barrio y familiares.

2 Plan Maestro de Espacio Público, Documento Técnico de Soporte, tomo 2, p. 19.

3 Ibíd. 